\title{
STUDY ON NOISES INFLUENCING THE ACCURACY OF CNC MACHINE STRAIGHTNESS MEASUREMENTS METHODS BASED ON BEAM POSITION DETECTION
}

\begin{abstract}
Fast, easy and accurate measurement of geometry of numerically controlled machines has always been challenging. With the use of a laser interferometer, it can be precise but it is complex. On the other side, the use of granite reference blocks is fairly easy yet quite limited for many machine types and sizes. Nowadays the improvements in technology open a new way - the instruments based on the detection of laser beam spot position. Those instruments are accurate and easy to use yet, comparing to the laser interferometers and granite blocks, they are sensitive to parameters of a laser source and to changing environmental conditions. In the paper, we analyse experimentally the influence of the type and parameters of laser source on the accuracy of straightness measurements. The most commonly met in practical applications a free space and fibre based laser sources are studied and compared. The influence of environmental conditions on measurement accuracy is also shown. Finally, the conclusions about optimal methods of improving the accuracy of beam position detection methods are drawn.
\end{abstract}

\section{INTRODUCTION}

Machines geometry measurements are important element of mechanical engineering. This is probably not going to change in any foreseeable future. The only difference between the past and the present are the tools used for this purpose. There exists a variety of possibilities, especially in the case of straightness measurements. Among various straightness measurement instruments recommended by the ISO committee in the major ISO230 series, laser interferometers became kind of a standard. It is because of their high accuracy at nanometre level, long travel of meters' range and direct traceability to the definition of the meter. However the accurate methods utilizing frequency type measurements require either complex optical setup (Wollaston prism measurements) or deliver specific or indirect results (angular optics measurements). The common point of the

\footnotetext{
${ }^{1}$ Faculty of Electronics, Wroclaw University of Science and Technology, Wroclaw, Poland

* E-mail: grzegorz.budzyn @pwr.edu.pl https://doi.org/10.36897/jme/127102
} 
laser interferometer use for straightness measurement is complexity and long duration of test procedure. In recent years a lot of effort has been taken by different teams to deliver a more time and cost efficient solution based on the laser beam position based measurements [1] also in two [2] or four degrees of freedom [3] measurement systems. The increasing precision of those methods is sufficient for machine testing with noticeably shorter measurement time as compared for example to laser interferometer based instruments.

The laser beam propagation through turbulent atmosphere have been evaluated in the literature extensively for many years both theoretically [4] and numerically [5, 6]. Main application areas where those effects are considered are astronomy, communication and various measurements [7]. Optical phenomena like air turbulence, beam scattering or beam absorption are caused by the variation of the refractive index along the path of the beam [8]. The changes in the refractive index are caused by the fluctuations of temperature which arise from the turbulent mixing of various thermal layers as proven experimentally in [9] or [10] or [11]. There have been described many different turbulence spectrum models for refraction fluctuations $[12,13]$ being a base for various numerical approaches $[14,15]$. Some tests were also described proving at least partial validity of those models for improvement of overall beam position stability in fibre [16], air [17] or vacuum [18] environments.

In this work there is delivered an analysis of two most important factors influencing the accuracy of such method: the laser beam drift caused by the air flow in the measurement path and the beam pointing stability of the laser source. We will show the ideas of improving the accuracy of the laser beam position based methods in practical applications.

\section{MEASUREMENT SETUPS}

The measurements analysed in this paper were conducted in the setup shown in Fig. 1 in the laboratory with air temperature within $22 \pm 1$ Celsius but with natural air flow. Forced airflow was used only for a part of measurement as stated in appropriate descriptions.

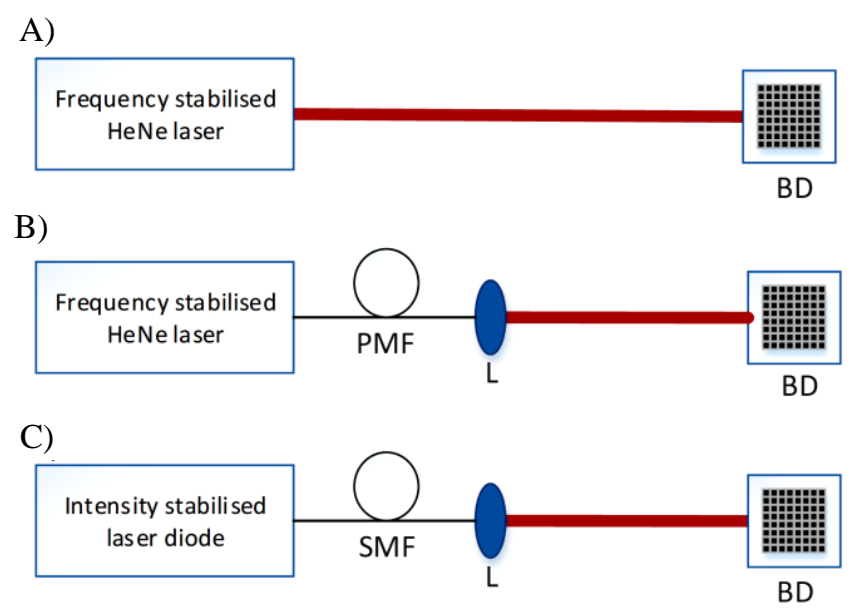

Fig. 1. Measurement circuits: A) - frequency stabilised 1mW He-Ne tube with collimated 5mm output beam; B) - the same laser head as in A but pigtailed to a PMF type fibre with collimated $5 \mathrm{~mm}$ output beam; C) - intensity stabilised $635 \mathrm{~nm}$ laser diode pigtailed to an SMF type fibre with collimated $5 \mathrm{~mm}$ output beam; 
The lasers with necessary optical components and the beam detector BD were mounted on stable concrete floor. The amplitude of the vibrations of the floor measured with the laser vibrometer was lower than $100 \mathrm{~nm}$.

There were used three popular but different laser sources. In the setup A) we used a free space frequency stabilised He-Ne laser, commonly utilized in industrial measurement systems. In the setup B) the same source was pigtailed to a polarisation maintaining fibre PMF. Such a configuration is also widely met in laboratory and industrial applications, where fibre delivery interferometry is required. In the last setup a semiconductor laser pigtailed with a single mode fibre SMF was exploited. Such a source cannot be used for frequency typed measurements because of very short coherence length but is quite suitable for amplitude type (e.g. beam position) measurements. Semiconductor type laser sources based are smaller, lighter and usually slightly cheaper compared to He-Ne sources. To both fibre sources beam collimators with high quality aspheric lens were attached resulting in an excellent quality $5 \mathrm{~mm}$ Gaussian beam profile. The same beam size was available at the output of the free space source.

As a position sensitive detector there are today commonly used three various solutions: a quadrant photodiode, a PSD detector and a camera module. The first solution is the simplest yet very sensitive to the beam shape. It is also very nonlinear outside of the central point. The PSD detectors are more linear than quadrant photodiodes and are not so sensitive on beam quality. The electronic part is also not very requiring. The most important disadvantages are low readout speed and still quite high linearity error. Besides, the position value obtained from this sensor is in a ready form giving no chances for digital improvements or amendments.

The last detector type, used in the experiments, is based on a CCD or CMOS camera sensor. Its advantages of high linearity, very good sensitivity and high configurability have only one serious disadvantage: high processing power requirements. This drawback can be today quite easily eliminated with the availability of a new breed of highly integrated and very fast microcontrollers. In the setup we were using a custom constructed CMOS sensor based module utilising a fast Cortex-M7 type microcontroller. This configuration allowed beam position detection in vertical and horizontal plane with detection rate of up to 1000 sample per second with measurement resolution of $0.1 \mu \mathrm{m}$ in the range of $10000 \times 10000 \mu \mathrm{m}$.

\section{MEASUREMENTS RESULTS}

From a large series of beam position measurements registered in the setups described in previous chapter, here are described only the most important results. During measurements the variations of laser beam position were registered at the maximum detector speed of $1000 \mathrm{sps}$ with no averaging. The measurement were conducted at distances between laser sources and beam detector from $1 \mathrm{~cm}$ up to $600 \mathrm{~cm}$ as noted later. In order to observe low frequency components each measurement was conducted for the duration of 30 minutes or more. The tests were conducted in the laboratory with a natural airflow unless otherwise noted. 
The influence of the air turbulence on the beam position value is clearly visible in Fig. 2, where the measurements recorded at two different distances between a PMF fibretype He-Ne laser and the beam detector BD are shown. The red line stands the beam position changes with detector placed only $1 \mathrm{~cm}$ from the laser output, while the blue line was used for measurement distance of $600 \mathrm{~cm}$. The difference is significant and is analysed in the following paragraphs. It is worth to notice that the amplitude of the noises was constant over the whole measurement time for both the short and long measurement distance. The small trend observed at $600 \mathrm{~cm}$ distance was probably due to the thermal drifts of fixtures of the PMF beam expander and the BD detector.

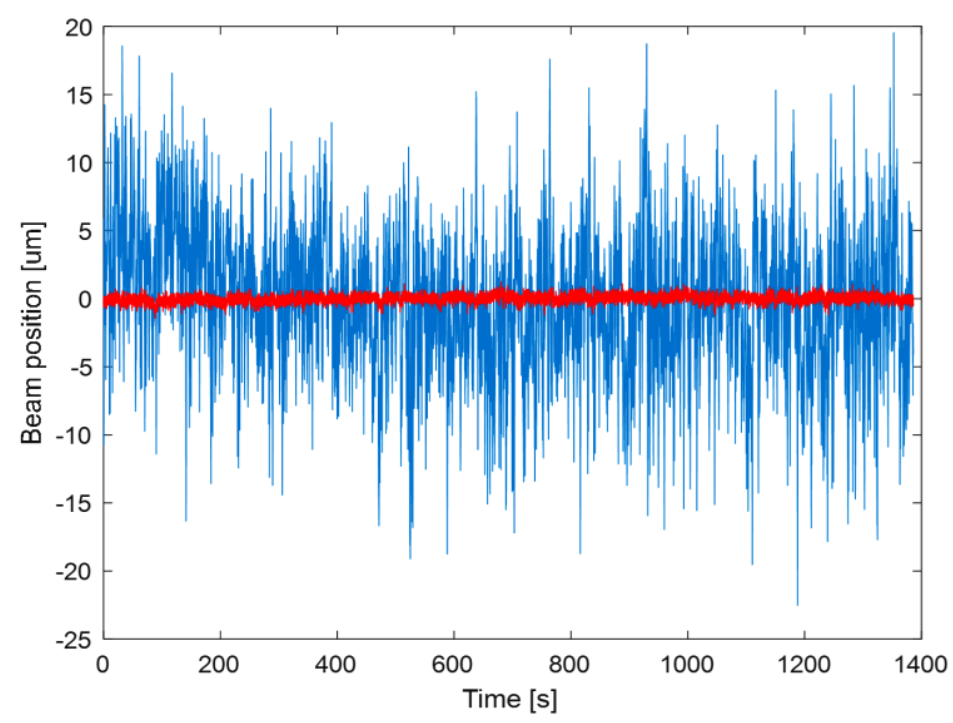

Fig. 2. Beam position fluctuations over time on a detector placed 0.01 meter (red) and $6 \mathrm{~m}$ (blue) from the PMF laser source. Pigtailed He-Ne source used

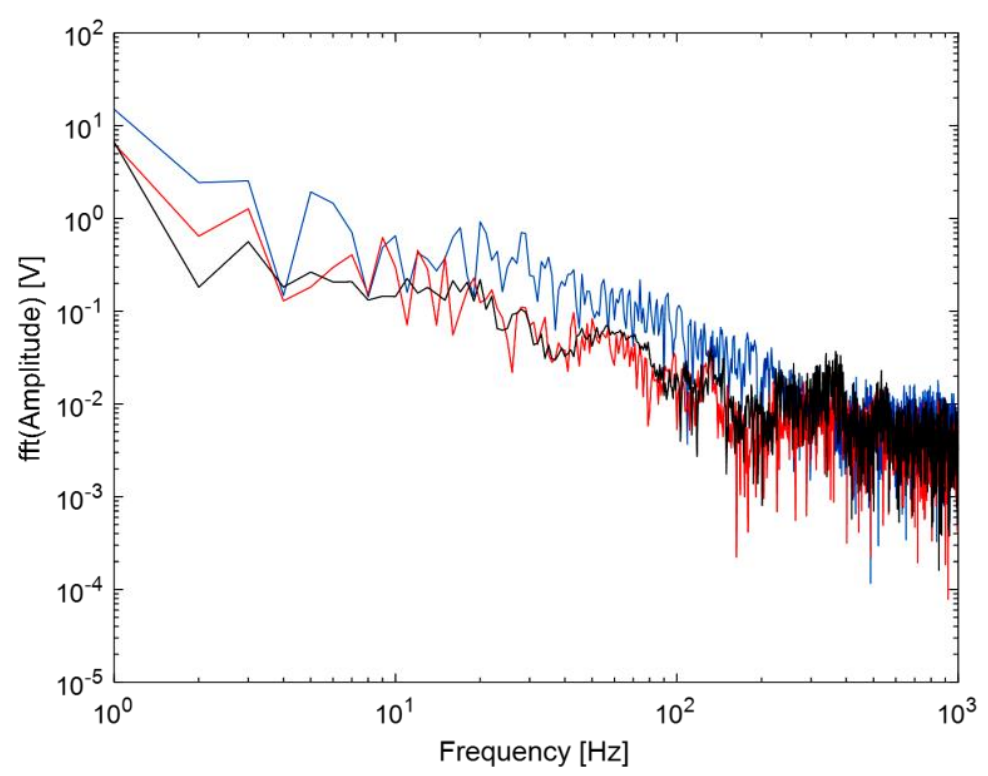

Fig. 3. Amplitude density spectrum for three different beam position measurements: black line represents fibre based source with detector $0.1 \mathrm{~m}$ from the source, red line represents fibre based He-Ne source with detector placed $2.0 \mathrm{~m}$ from the source, blue line represents free space source with detector placed $6.0 \mathrm{~m}$ from the source 
The SNR of real signals can usually be improved with a signal filtering or signal averaging techniques. In order to choose the most optimal filtering technique we analysed the beam position signal in the frequency domain. As shown in Fig. 3 the spectral density of the beam position resembles $1 / f$ or pink noise especially for smaller distances between laser and BD. A comparison was made between the a low-pass FIR filter and a simple signal smoothing. The simpler technique, requiring just a fraction of the processing power of a FIR filter, proved more viable - see Fig. 4. Both filtering versions were not able to filter out near DC noise from the signal as could be expected for $1 / f$ type signals still the observed overall SNR improvement (Fig. 5) is very significant. The cut-off frequency of the filter was set to $1 \mathrm{~Hz}$, which seems be the sufficient value for usual machine geometry measurement cases. Such a filter helped to reduce peak-to-peak noises by about a factor of 4 .

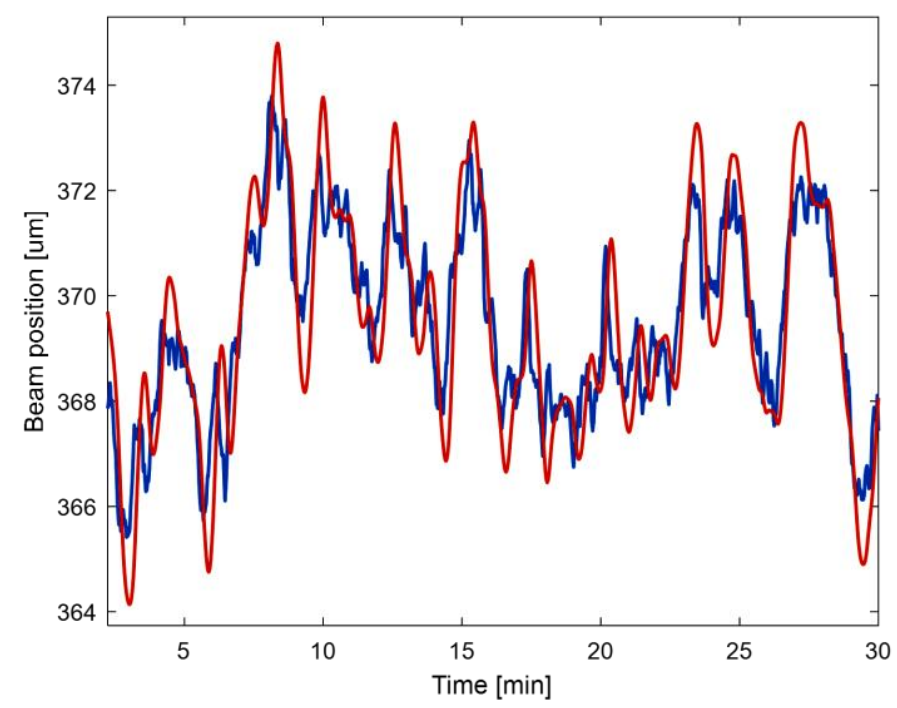

Fig. 4. Difference between the use of low-pass FIR filter (red) and signal smoothing (blue) on the quality of final beam position signal.

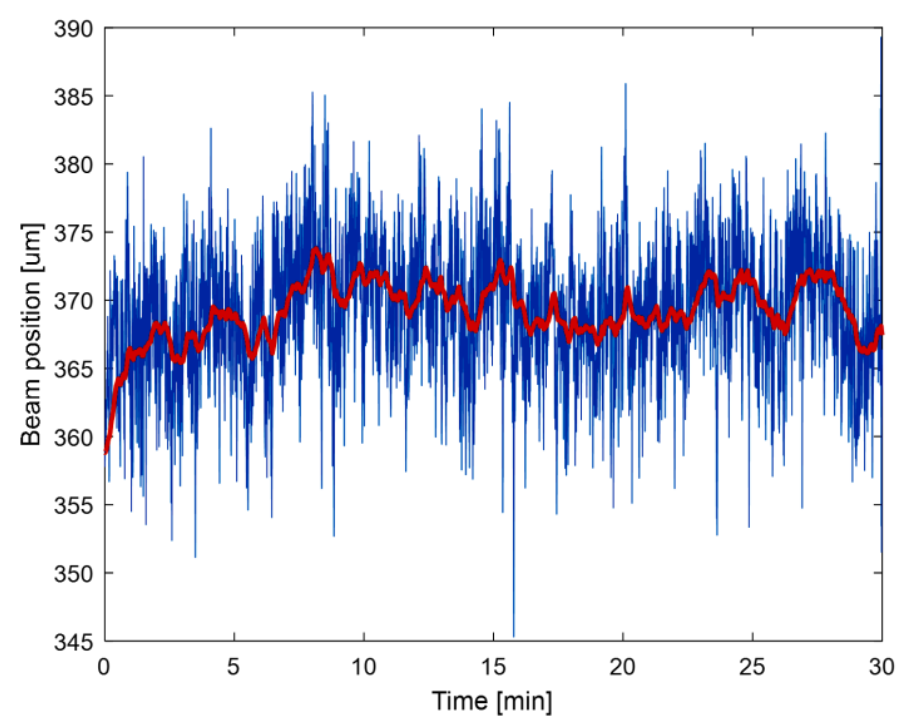

Fig. 5. Influence of beam position signal averaging (cut off frequency of $1 \mathrm{~Hz}$ ) on the quality of output results 


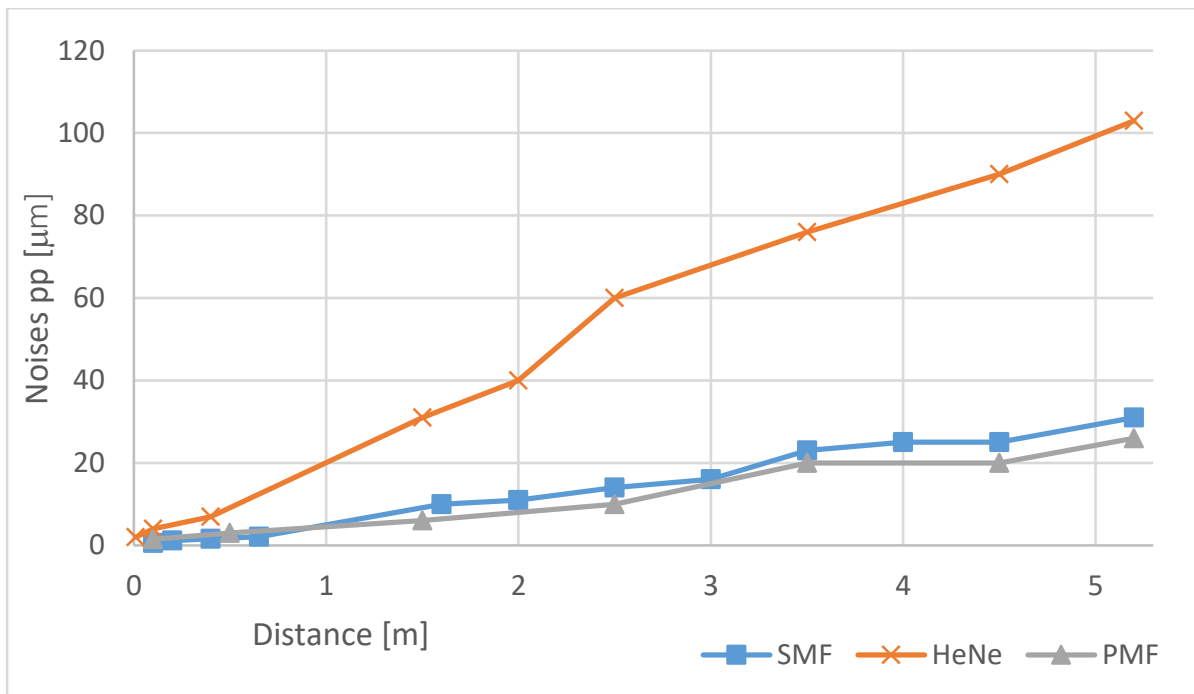

Fig. 6. Amplitude of beam position fluctuations as a function of distance between laser source and beam detector for three different laser sources: SMF - single mode fibre pigtailed laser diode, He-Ne - frequency stabilised He-Ne tube, $\mathrm{PMF}$ - polarisation fibre pigtailed frequency stabilised He-Ne tube

The stability of all three laser sources was compared over a distance from $1 \mathrm{~cm}$ to $520 \mathrm{~cm}$. The non-averaged noises were shown in Fig. 6. The observed dependence is linear for all sources. There is noticeable difference between free space and both fibre solutions. The PMF fibre type He-Ne source offers significantly lower position noises as compared to the free space solution. From this fact it can be estimated that the pointing stability of a free space frequency stabilised He-Ne source is around $20 \mu \mathrm{rad}$ which is better than the catalogue value for the tube used (LGR7655 - $1 \mathrm{mrad}$ ). The non-averaged beam position noises for both fibre sources were scaling with the distance between laser and detector with the magnitude of around $5.5 \mu \mathrm{m}$ per each meter. The noises for free space laser were around four times larger.

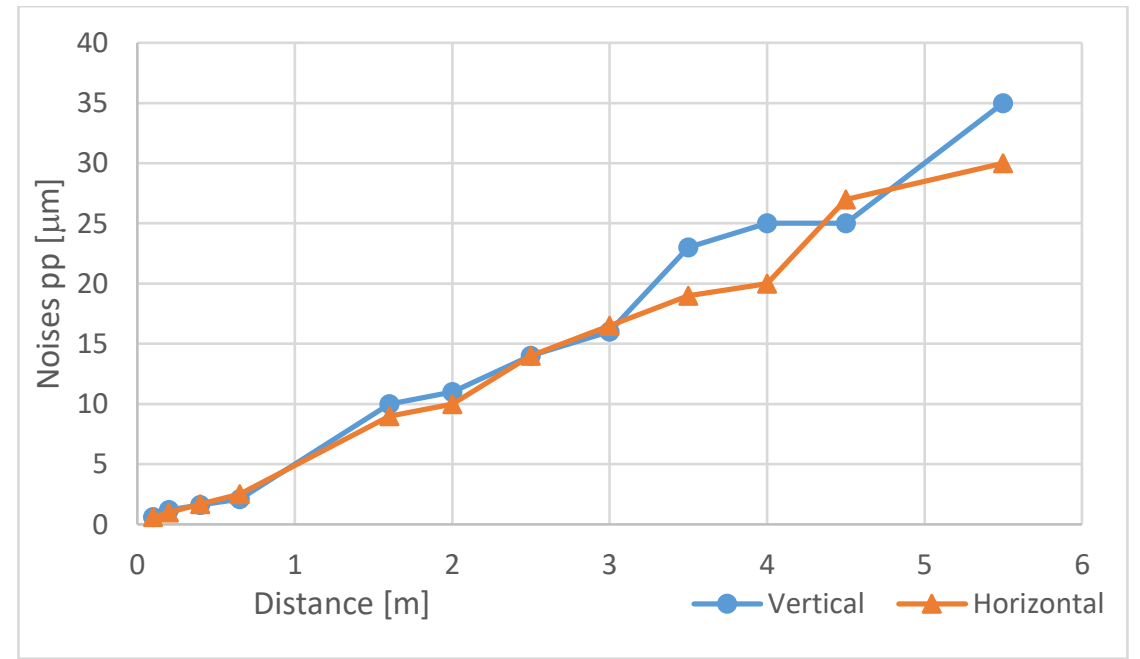

Fig. 7. Amplitude of beam position fluctuations (non-averaged) as a function of distance measured in vertical and horizontal beam plane 
We did not notice any difference in the amplitude of position noises in vertical and horizontal planes as it is visible in Fig. 7. The chart would be different in situations when the airflow is forced both advertently (e.g. because of a running air-conditioning) or inadvertently (e.g. a draft). Such tests were performed as well and the results of nonaveraged and averaged fluctuations are shown in Fig. 8. The forced air flow increases the high frequency noises yet not influencing (in both positive and negative manner) the useful DC and near DC-range. The filtered position when the airflow was forced and when it was natural was similar. The reverse situation, when the beam was shielded from the airflow in narrow tube is shown in Fig. 9.

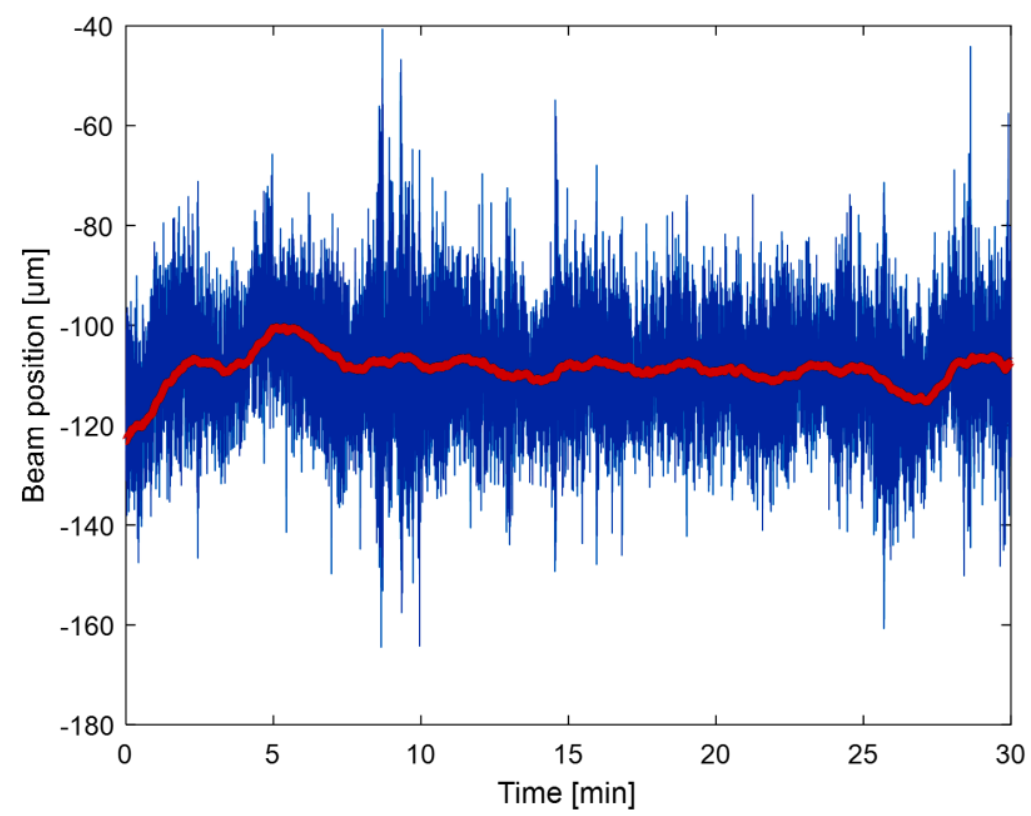

Fig. 8. Fluctuations of beam position on the distance of $5.2 \mathrm{~m}$ with the artificial air flow perpendicular to beam direction caused by a running fan. The blue line shows the non-averaged signal, the red one - averaged with signal smoothing algorithm

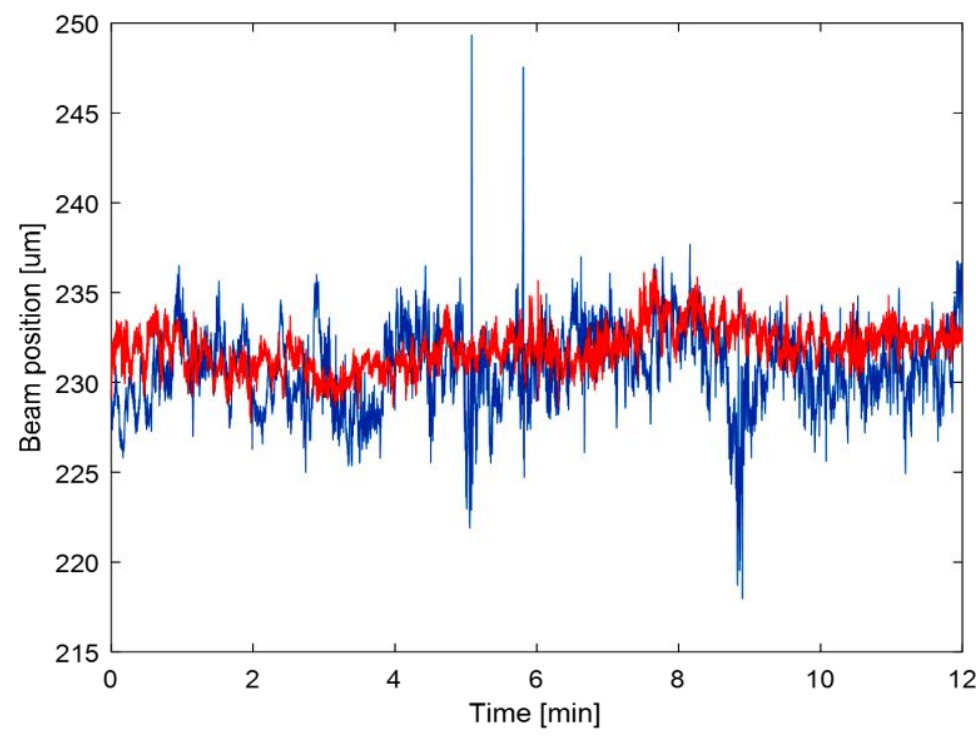

Fig. 9. Fluctuations of beam position on the distance of $2.0 \mathrm{~m}$ in the open air (blue) or in the narrow tube (red) 
Although the observed non-averaged noise was much smaller than the noise especially in the forced airflow case but after averaging the output signal fluctuations were in all cases similar. The conclusion can be drawn that unless a turbulent airflow is present, the beam shielding may be avoided in normal measurement conditions.

Finally the beam position fluctuations from all three tested laser sources were averaged with $1 \mathrm{~Hz}$ cut-off exponential smoothing filter over a distance from $1 \mathrm{~cm}$ to $520 \mathrm{~cm}$. The observed dependence is still linear (compare Fig. 6 with Fig. 10) scaling with the distance of about $2 \mu \mathrm{m}$ per meter for both fibre sources and $3.5 \mu \mathrm{m}$ per meter for free space He-Ne tube. The improvement is especially viable for free space He-Ne source. The observed beam position noises define the accuracy limit of beam position error measurements in practical applications. If the required accuracy should be better than the values presented in Fig. 10 then the standard frequency type methods (like Wollaston prism method) have to be utilized.

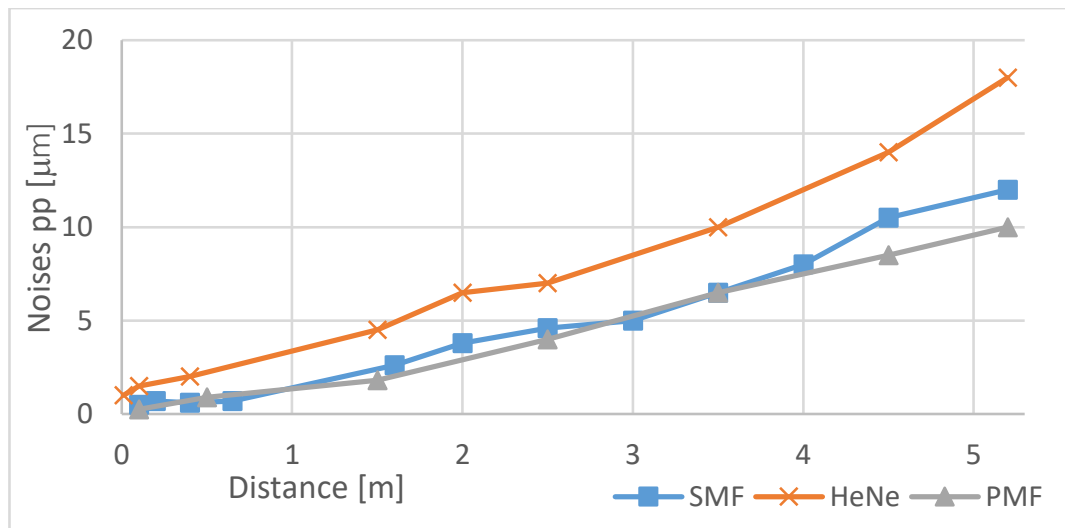

Fig. 10. Averaged amplitude of beam position fluctuations as a function of distance between laser source and beam detector for three different laser sources: SMF - single mode fibre pigtailed laser diode, $\mathrm{He}-\mathrm{Ne}$ - frequency stabilised $\mathrm{HeNe}$ tube, PMF - polarisation fibre pigtailed frequency stabilised $\mathrm{He}-\mathrm{Ne}$ tube

The results shown in Fig. 10 can be used also for estimation of the beam position uncertainty caused by the beam detector BD. Because the same detector was used during all conducted measurements thus the beam position uncertainty is not higher than the lowest beam position fluctuation registered for any source and any distance. In our case the measurement uncertainty was lower than $0.5 \mu \mathrm{m}$.

\section{SUMMARY AND CONCLUSIONS}

It is clearly seen from the presented measurements that the technique of beam position based machine straightness measurements is burdened with two major factors - the fluctuations of air in the measurement path and the beam pointing stability of the laser source. The influence of the first element can only be averaged out. Beam path shielding is 
not giving expected improvements and is very impractical. Imposing a forced air flow, as suggested in some sources, also does not bring visible improvements and is deteriorating the values if the imposed flow is turbulent. In the case of the laser source choice a single mode fibre based instruments seem to be more optimal. The use of free space HeNe laser defines the accuracy limit to be only slightly better than $4 \mu \mathrm{m}$ per each meter of distance between the laser and the detector.

\section{REFERENCES}

[1] CHEN B., CHENG L., YAN L., ZHANG E., LOU Y., 2017, A Heterodyne Straightness and Displacement Measuring Interferometer with Laser Beam Drift Compensation for Long-Travel Linear Stage Metrology, Rev. Sci. Instrum., 88, 035114.

[2] KUANG C., HONG E., FENG Q., ZHANG B., ZHANG Z., 2007, A Novel Method to Enhance the Sensitivity for Two-Degrees-of-Freedom Straightness Measurement, Meas. Sci. Technol., 18, 3795-3800.

[3] YUBIN H., KUANG C.F, WEI S., SHUJITE L., 2018, Low Cost, Compact 4-DOF Measurement System with Active Compensation of Beam Angular Drift Error, Optics Express, 26/13, 17185-17198.

[4] CONSORTINI A., RONCHI L., STEFANUTTI L., 1970, Investigation of Atmospheric Turbulence by Narrow Laser Beams, Appl. Opt., 9, 2543-2547.

[5] YAHE R.Z., LAST I., 1992, Numerical Simulation of Laser Beam Propagation in Three-Dimensional Random Media: Beam Splitting and Patch Formation, Waves in Random Media, 2, 81-98.

[6] CAI Y., 2006, Propagation of Various Flat-Topped Beams in a Turbulent Atmosphere , J. OPT. A-PURE, 8, 537-545.

[7] ZHAO W., QIU L., FENG Z., LI C., 2006, Laser Beam Alignment by Fast Feedback Control of Both Linear and Angular Drifts, Optik, 117, 505.

[8] MURTY S.C.C., 1979, Laser Beam Propagation in Atmospheric Turbulence, Prec. Indian Acad. Sci., C 2, Part 2, May, 179-195.

[9] YUSHENG Z., 2015, Experimental Study of Laser Beam Drift, International Conference on Optoelectronics and Microelectronics (ICOM).

[10] KLEMM K., PIESZYNSKI K., ROZNIAKOWSKI K., 2007, Examination of Air Density Fluctuations with the Aid of Laser Beam, Optica Applicata, XXXVII, 3.

[11] LI Y., QI J., CHEN F., 2017, Propagation Quality of Laser Diode Beam in Anisotropic Non-Kolmogorov Atmospheric Turbulence, Acta Optica Sinica, 37/7.

[12] TATARSKI V.I., 1971, Wave Propagation in Turbulent Medium, (Springfield, Virginia: National Technical Information Service).

[13] KERR J.R., DUNPHY J.R., 1973, Scintillation Measurements for Large Integrated-Path Turbulence, J. Opt. Soc. Am., 63.

[14] MAHDIEH M.H., 2008, Numerical Approach to Laser Beam Propagation Through Turbulent Atmosphere and Evaluation of Beam Quality Factor, Optics Communications, 281, 3395-3402.

[15] LI J., WEI H., YAN LI Y., 2019, Beam Drift Reduction by Straightness Measurement Based on a Digital Optical Phase Conjugation, Applied Optics, 58/27, 7636-7642.

[16] ZHAO W., TAN J., QIU L., et al., 2005, Enhancing Laser Beam Directional Stability by Single-Mode Optical Fiber and Feedback Control of Drifts, Rev. Sci. Instrum., 76, 036101.

[17] GRAYSON K., 2017, Beam Stability and Warm-Up Effects of Nd: YAG Lasers Used in Particle Image Velocimetry, Meas. Sci. Technol., 28, 065301.

[18] STERN G., 2014, Experiments of Laser Pointing Stability in Air and in Vacuum to Validate Micrometric Positioning Sensor, 5th International Particle Accelerator Conference IPAC, Dresden, Germany. 